\title{
Crystal structure of dekalanthanum tetrabromide pentaaluminide, $\mathrm{La}_{10} \mathrm{Br}_{4} \mathrm{Al}_{5}$
}

\author{
Hj. Mattausch*, O. Oeckler and A. Simon
}

Max-Planck-Institut für Festkörperforschung, Heisenbergstraße 1, D-70569 Stuttgart, Germany

Received May 19, 2003, accepted and available on-line July 23, 2003; CSD-No. 409704

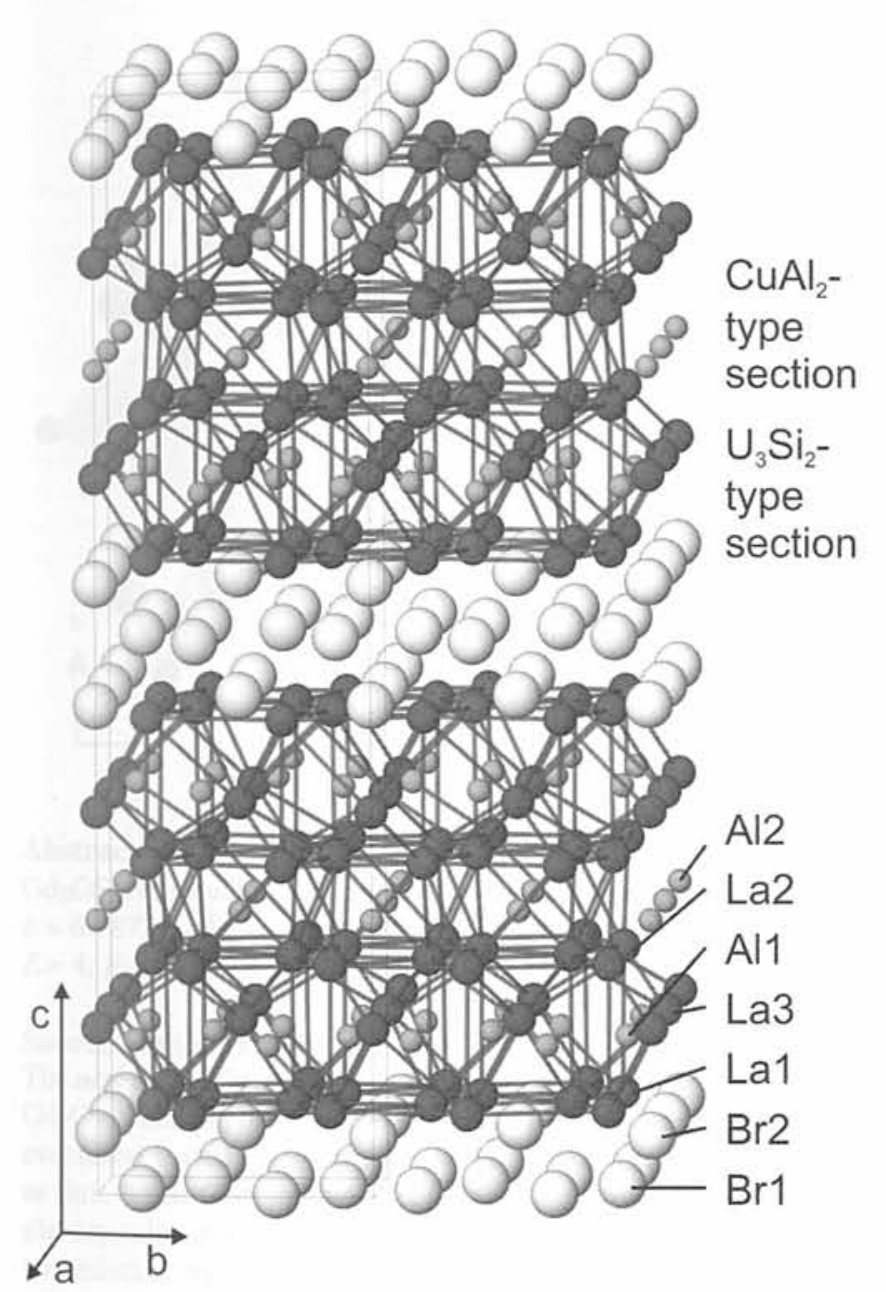

Abstract

$\mathrm{Al}_{5} \mathrm{Br}_{4} \mathrm{La}_{10}$, tetragonal, $I 4 / m c m$ (No. 140$), a=8.2713(8) \AA$, $c=32.835(5) \AA, V=2246.4 \AA^{3}, Z=4, R_{\mathrm{gt}}(F)=0.027$, $w R_{\text {ref }}\left(F^{2}\right)=0.058, T=293 \mathrm{~K}$.

\section{Source of material}

$\mathrm{La}_{10} \mathrm{Br}_{4} \mathrm{Al}_{5}$ is prepared by heating stoichiometric amounts of $\mathrm{LaBr}_{3}, \mathrm{La}$ and $\mathrm{Al}$ under $\mathrm{Ar}$ atmosphere in sealed Ta-capsules at $1125 \mathrm{~K}$ for 7 days.

\section{Discussion}

$\mathrm{La}_{10} \mathrm{Br}_{4} \mathrm{Al}_{5}$ is isotypic with $\mathrm{La}_{10} \mathrm{X}_{4} \mathrm{Ga}_{5}(\mathrm{X}=\mathrm{Cl}, \mathrm{Br})$ and $\mathrm{Ce}_{10} \mathrm{Cl}_{4} \mathrm{Ga}_{5}[1,2]$. The crystal structure consists of $\mathrm{La}-\mathrm{Al}$ slabs separated by layers of $\mathrm{Br}$ ions. The two types of the metal layer topology correspond to a planar section of the $\mathrm{CuAl}_{2}$-type structure condensed with a 2-dimensional section of the $\mathrm{U}_{3} \mathrm{Si}_{2}$ - structure type. The distances $\mathrm{La}-\mathrm{La}$ range from 4.022(1) $\AA$ to 4.104(1) $\AA$, $\mathrm{La}-\mathrm{Br}$ from 3.110 (1) $\AA$ to 3.170 (1) $\AA$, and $\mathrm{La}-\mathrm{Al}$ from $3.210(2) \AA$ to 3.350 (2) $\AA$.

Table 1. Data collection and handling.

\section{Crystal:}

Wavelength:

$\mu$ :

Diffractometer, scan mode:

$2 \theta_{\max }$ :

$N(h k l)_{\text {measured, }} N(h k l)_{\text {unique: }}$

Criterion for $I_{\mathrm{obs}}, N(h k l) \mathrm{gt}$ :

$N(\text { param })_{\text {refined: }}$

Programs: silver platelet, size $0.06 \times 0.20 \times 0.20 \mathrm{~mm}$ Mo $K_{\alpha}$ radiation $(0.71073 \AA)$ $258.45 \mathrm{~cm}^{-1}$

Stoe IPDS, $\omega / \theta$

$57.98^{\circ}$

6957,822

$I_{\text {obs }}>2 \sigma\left(I_{\text {obs }}\right), 794$

32

SHELXL-97 [3], ATOMS [4]

Table 2. Atomic coordinates and displacement parameters (in $\AA^{2}$ ).

\begin{tabular}{lllllllllll}
\hline Atom & Site & $x$ & $y$ & $z$ & $U_{11}$ & $U_{22}$ & $U_{33}$ & $U_{12}$ & $U_{13}$ & $U_{23}$ \\
\hline $\mathrm{La}(1)$ & $16 l$ & $0.67194(3)$ & $x+1 / 2$ & $0.42846(1)$ & $0.0127(2)$ & $U_{11}$ & $0.0166(2)$ & $0.0010(1)$ & $-0.00181(9)$ & $U_{13}$ \\
$\mathrm{La}(2)$ & $16 l$ & $0.66757(4)$ & $x+1 / 2$ & $0.29608(1)$ & $0.0157(2)$ & $U_{11}$ & $0.0189(2)$ & $0.0007(1)$ & $0.0008(1)$ & $U_{13}$ \\
$\mathrm{La}(3)$ & $8 f$ & $1 / 2$ & $1 / 2$ & $0.34817(2)$ & $0.0183(2)$ & $U_{11}$ & $0.0150(3)$ & 0 & 0 & 0 \\
$\mathrm{Br}(1)$ & $8 f$ & 0 & 0 & $0.94471(3)$ & $0.0128(3)$ & $U_{11}$ & $0.0177(4)$ & 0 & 0 & 0 \\
$\operatorname{Br}(2)$ & $8 h$ & $0.85785(9)$ & $x+1 / 2$ & $1 / 2$ & $0.0188(3)$ & $U_{11}$ & $0.0211(5)$ & $-0.0028(4)$ & 0 & 0 \\
$\mathrm{Al}(1)$ & $16 l$ & $0.3814(2)$ & $-x+1 / 2$ & $0.36506(6)$ & $0.0133(6)$ & $U_{11}$ & $0.015(1)$ & $0.0003(8)$ & $0.0001(5)$ & $-U_{13}$ \\
$\mathrm{Al}(2)$ & $4 a$ & $1 / 2$ & $1 / 2$ & $1 / 4$ & $0.015(1)$ & $U_{11}$ & $0.015(2)$ & 0 & 0 & 0 \\
\hline
\end{tabular}

\footnotetext{
* Correspondence author (e-mail: Hj.Mattausch@fkf.mpg.de)
} 


\section{References}

1. Zheng, C.; Simon, A.; Mattausch, Hj.: Reduced lanthanum halides with $\mathrm{Ga}$ as interstitials: $\mathrm{La}_{10} \mathrm{Cl}_{4} \mathrm{Ga}_{4}, \mathrm{La}_{10} \mathrm{Br}_{4} \mathrm{Ga}_{5}$ and $\mathrm{La}_{3} \mathrm{Br}_{3} \mathrm{Ga}$. J. Alloys Compds. 338 (2002) 165-172.

2. Zheng, C.; Oeckler, O.; Mattausch, $\mathrm{Hj}$.; Simon, A.: $\mathrm{Ce}_{10} \mathrm{Cl}_{4} \mathrm{Ga}_{5}$ and $\mathrm{Ln}_{3} \mathrm{ClGa}_{4}(\mathrm{Ln}=\mathrm{La}, \mathrm{Ce})$ : Reduced Halides or Oxidized Intermetallics? Inorg. Chem. 42 (2003) 3130-3135.

3. Sheldrick, G. M.: SHELXL-97. Program for refining crystal structures. University of Göttingen, Germany 1997.

4. Dowty, E.: Atoms 5.0, A Complete Program for Displaying Atomic Structures. By Shape Software, Kingsport, TN 37663, USA 1999. 